\title{
Bifurcation Analysis of a Prey-Predator Coevolution Model
}

Fabio Dercole (dercole@elet.polimi.it)

Jean-Olivier Irisson (irisson@horus.ens.fr)

Sergio Rinaldi (rinaldi@elet.polimi.it)

\section{Approved by}

Ulf Dieckmann (dieckman@iiasa.ac.at)

Project Leader, Adaptive Dynamics Network

December 2002 


\section{IIASA STUDIES IN ADAPTIVE DYNAMICS No. 70}

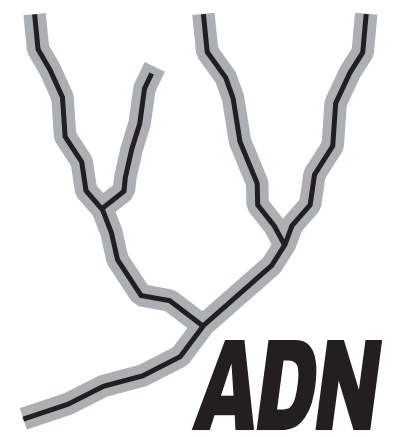

The Adaptive Dynamics Network at IIASA fosters the development of new mathematical and conceptual techniques for understanding the evolution of complex adaptive systems.

Focusing on these long-term implications of adaptive processes in systems of limited growth, the Adaptive Dynamics Network brings together scientists and institutions from around the world with IIASA acting as the central node.

Scientific progress within the network is collected in the IIASA Studies in Adaptive Dynamics series.

No. 1 Metz JAJ, Geritz SAH, Meszéna G, Jacobs FJA, van Heerwaarden JS: Adaptive Dynamics: A Geometrical Study of the Consequences of Nearly Faithful Reproduction. IIASA Working Paper WP-95-099 (1995). van Strien SJ, Verduyn Lunel SM (eds): Stochastic and Spatial Structures of Dynamical Systems, Proceedings of the Royal Dutch Academy of Science (KNAW Verhandelingen), North Holland, Amsterdam, pp. 183-231 (1996).

No. 2 Dieckmann U, Law R: The Dynamical Theory of Coevolution: A Derivation from Stochastic Ecological Processes. IIASA Working Paper WP-96-001 (1996). Journal of Mathematical Biology 34:579-612 (1996).

No. 3 Dieckmann U, Marrow P, Law R: Evolutionary Cycling of Predator-PreyInteractions: Population Dynamics and the Red Queen. IIASA Preprint (1995). Journal of Theoretical Biology 176:91-102 (1995).

No. 4 Marrow P, Dieckmann U, Law R: Evolutionary Dynamics of Predator-Prey Systems: An Ecological Perspective. IIASA Working Paper WP-96-002 (1996). Journal of Mathematical Biology 34:556-578 (1996).

No. 5 Law R, Marrow P, Dieckmann U: On Evolution under Asymmetric Competition. IIASA Working Paper WP-96-003 (1996). Evolutionary Ecology 11:485-501 (1997).

No. 6 Metz JAJ, Mylius SD, Diekmann O: When Does Evolution Optimize? On the Relation Between Types of Density Dependence and Evolutionarily Stable Life History Parameters. IIASA Working Paper WP-96-004 (1996).

No. 7 Ferrière R, Gatto M: Lyapunov Exponents and the Mathematics of Invasion in Oscillatory or Chaotic Populations. Theoretical Population Biology 48:126-171 (1995).

No. 8 Ferrière R, Fox GA: Chaos and Evolution. IIASA Preprint (1996). Trends in Ecology and Evolution 10:480485 (1995).

No. 9 Ferrière R, Michod RE: The Evolution of Cooperation in Spatially Heterogeneous Populations. IIASA Working Paper WP-96-029 (1996). The American Naturalist 147:692717 (1996).

No. 10 van Dooren TJM, Metz JAJ: Delayed Maturation in Temporally Structured Populations with Non-Equilibrium Dynamics. IIASA Working Paper WP-96-070 (1996). Journal of Evolutionary Biology 11:41-62 (1998).
No. 11 Geritz SAH, Metz JAJ, Kisdi É, Meszéna G: The Dynamics of Adaptation and Evolutionary Branching. IIASA Working Paper WP-96-077 (1996). Physical Review Letters 78:2024-2027 (1997).

No. 12 Geritz SAH, Kisdi É, Meszéna G, Metz JAJ: Evolutionary Singular Strategies and the Adaptive Growth and Branching of the Evolutionary Tree. IIASA Working Paper WP-96-114 (1996). Evolutionary Ecology 12:35-57 (1998).

No. 13 Heino M, Metz JAJ, Kaitala V: Evolution of Mixed Maturation Strategies in Semelparous Life-Histories: The Crucial Role of Dimensionality of Feedback Environment. IIASA Working Paper WP-96-126 (1996). Philosophical Transactions of the Royal Society of London Series B 352:1647-1655 (1997).

No. 14 Dieckmann U: Can Adaptive Dynamics Invade? IIASA Working Paper WP-96-152 (1996). Trends in Ecology and Evolution 12:128-131 (1997).

No. 15 Meszéna G, Czibula I, Geritz SAH: Adaptive Dynamics in a 2-Patch Environment: A Simple Model for Allopatric and Parapatric Speciation. IIASA Interim Report IR-97-001 (1997). Journal of Biological Systems 5:265-284 (1997).

No. 16 Heino M, Metz JAJ, Kaitala V: The Enigma of Frequency-Dependent Selection. IIASA Interim Report IR97-061 (1997). Trends in Ecology and Evolution 13:367-370 (1998).

No. 17 Heino M: Management of Evolving Fish Stocks. IIASA Interim Report IR-97-062 (1997). Canadian Journal of Fisheries and Aquatic Sciences 55:1971-1982 (1998).

No. 18 Heino M: Evolution of Mixed Reproductive Strategies in Simple Life-History Models. IIASA Interim Report IR-97063 (1997).

No. 19 Geritz SAH, van der Meijden E, Metz JAJ: Evolutionary Dynamics of Seed Size and Seedling Competitive Ability. IIASA Interim Report IR-97-071 (1997). Theoretical Population Biology 55:324-343 (1999).

No. 20 Galis F, Metz JAJ: Why Are There So Many Cichlid Species? On the Interplay of Speciation and Adaptive Radiation. IIASA Interim Report IR-97-072 (1997). Trends in Ecology and Evolution 13:1-2 (1998). 
No. 21 Boerlijst MC, Nowak MA, Sigmund K: Equal Pay for all Prisoners/ The Logic of Contrition. IIASA Interim Report IR-97-073 (1997). American Mathematical Society Monthly 104:303-307 (1997). Journal of Theoretical Biology 185:281-293 (1997).

No. 22 Law R, Dieckmann U: Symbiosis Without Mutualism and the Merger of Lineages in Evolution. IIASA Interim Report IR-97-074 (1997). Proceedings of the Royal Society of London Series B 265:1245-1253 (1998).

No. 23 Klinkhamer PGL, de Jong TJ, Metz JAJ: Sex and Size in Cosexual Plants. IIASA Interim Report IR-97-078 (1997). Trends in Ecology and Evolution 12:260-265 (1997).

No. 24 Fontana W, Schuster P: Shaping Space: The Possible and the Attainable in RNA Genotype-Phenotype Mapping. IIASA Interim Report IR-98-004 (1998). Journal of Theoretical Biology 194:491-515 (1998).

No. 25 Kisdi É, Geritz SAH: Adaptive Dynamics in Allele Space: Evolution of Genetic Polymorphism by Small Mutations in a Heterogeneous Environment. IIASA Interim Report IR-98-038 (1998). Evolution 53:993-1008 (1999).

No. 26 Fontana W, Schuster P: Continuity in Evolution: On the Nature of Transitions. IIASA Interim Report IR-98-039 (1998). Science 280:1451-1455 (1998).

No. 27 Nowak MA, Sigmund K: Evolution of Indirect Reciprocity by Image Scoring/ The Dynamics of Indirect Reciprocity. IIASA Interim Report IR-98-040 (1998). Nature 393:573-577 (1998). Journal of Theoretical Biology 194:561574 (1998).

No. 28 Kisdi É: Evolutionary Branching Under Asymmetric Competition. IIASA Interim Report IR-98-045 (1998). Journal of Theoretical Biology 197:149-162 (1999).

No. 29 Berger U: Best Response Adaptation for Role Games. IIASA Interim Report IR-98-086 (1998).

No. 30 van Dooren TJM: The Evolutionary Ecology of Dominance-Recessivity. IIASA Interim Report IR-98-096 (1998). Journal of Theoretical Biology 198:519-532 (1999).

No. 31 Dieckmann U, O'Hara B, Weisser W: The Evolutionary Ecology of Dispersal. IIASA Interim Report IR-98-108 (1998). Trends in Ecology and Evolution 14:88-90 (1999).

No. 32 Sigmund K: Complex Adaptive Systems and the Evolution of Reciprocation. IIASA Interim Report IR-98-100 (1998). Ecosystems 1:444-448 (1998).

No. 33 Posch M, Pichler A, Sigmund K: The Efficiency of Adapting Aspiration Levels. IIASA Interim Report IR-98103 (1998). Proceedings of the Royal Society London Series B 266:1427-1435 (1999).

No. 34 Mathias A, Kisdi É: Evolutionary Branching and Coexistence of Germination Strategies. IIASA Interim Report IR-99-014 (1999).

No. 35 Dieckmann U, Doebeli M: On the Origin of Species by Sympatric Speciation. IIASA Interim Report IR-99-013 (1999). Nature 400:354-357 (1999).

No. 36 Metz JAJ, Gyllenberg M: How Should We Define Fitness in Structured Metapopulation Models? Including an Application to the Calculation of Evolutionarily Stable Dispersal Strategies. IIASA Interim Report IR-99-019 (1999). Proceedings of the Royal Society of London Series B 268:499$508(2001)$
No. 37 Gyllenberg M, Metz JAJ: On Fitness in Structured Metapopulations. IIASA Interim Report IR-99-037 (1999). Journal of Mathematical Biology 43:545-560 (2001).

No. 38 Meszéna G, Metz JAJ: Species Diversity and Population Regulation: The Importance of Environmental Feedback Dimensionality. IIASA Interim Report IR-99-045 (1999).

No. 39 Kisdi É, Geritz SAH: Evolutionary Branching and Sympatric Speciation in Diploid Populations. IIASA Interim Report IR-99-048 (1999).

No. 40 Ylikarjula J, Heino M, Dieckmann U: Ecology and Adaptation of Stunted Growth in Fish. IIASA Interim Report IR-99-050 (1999). Evolutionary Ecology 13:433-453 (1999).

No. 41 Nowak MA, Sigmund K: Games on Grids. IIASA Interim Report IR-99-038 (1999). Dieckmann U, Law R, Metz JAJ (eds): The Geometry of Ecological Interactions: Simplifying Spatial Complexity, Cambridge University Press, Cambridge, UK, pp. 135-150 (2000).

No. 42 Ferrière R, Michod RE: Wave Patterns in Spatial Games and the Evolution of Cooperation. IIASA Interim Report IR-99-041 (1999). Dieckmann U, Law R, Metz JAJ (eds): The Geometry of Ecological Interactions: Simplifying Spatial Complexity, Cambridge University Press, Cambridge, UK, pp. 318-332 (2000).

No. 43 Kisdi É, Jacobs FJA, Geritz SAH: Red Queen Evolution by Cycles of Evolutionary Branching and Extinction. IIASA Interim Report IR-00-030 (2000). Selection 2:161176 (2001).

No. 44 Meszéna G, Kisdi É, Dieckmann U, Geritz SAH, Metz JAJ: Evolutionary Optimisation Models and Matrix Games in the Unified Perspective of Adaptive Dynamics. IIASA Interim Report IR-00-039 (2000). Selection 2:193-210 (2001).

No. 45 Parvinen K, Dieckmann U, Gyllenberg M, Metz JAJ: Evolution of Dispersal in Metapopulations with Local Density Dependence and Demographic Stochasticity. IIASA Interim Report IR-00-035 (2000).

No. 46 Doebeli M, Dieckmann U: Evolutionary Branching and Sympatric Speciation Caused by Different Types of Ecological Interactions. IIASA Interim Report IR-00-040 (2000). The American Naturalist 156:S77-S101 (2000).

No. 47 Heino M, Hanski I: Evolution of Migration Rate in a Spatially Realistic Metapopulation Model. IIASA Interim Report IR-00-044 (2000). The American Naturalist 157:495511 (2001).

No. 48 Gyllenberg M, Parvinen K, Dieckmann U: Evolutionary Suicide and Evolution of Dispersal in Structured Metapopulations. IIASA Interim Report IR-00-056 (2000). Journal of Mathematical Biology 45:79-105 (2002).

No. 49 van Dooren TJM: The Evolutionary Dynamics of Direct Phenotypic Overdominance: Emergence Possible, Loss Probable. IIASA Interim Report IR-00-048 (2000). Evolution 54: 1899-1914 (2000).

No. 50 Nowak MA, Page KM, Sigmund K: Fairness Versus Reason in the Ultimatum Game. IIASA Interim Report IR00-57 (2000). Science 289:1773-1775 (2000).

No. 51 de Feo O, Ferrière R: Bifurcation Analysis of Population Invasion: On-Off Intermittency and Basin Riddling. IIASA Interim Report IR-00-074 (2000). International Journal of Bifurcation and Chaos 10:443-452 (2000). 
No. 52 Heino M, Laaka-Lindberg S: Clonal Dynamics and Evolution of Dormancy in the Leafy Hepatic Lophozia Silvicola. IIASA Interim Report IR-01-018 (2001). Oikos 94:525-532 (2001).

No. 53 Sigmund K, Hauert C, Nowak MA: Reward and Punishment in Minigames. IIASA Interim Report IR-01-031 (2001). Proceedings of the National Academy of Sciences of the USA 98:10757-10762(2001).

No. 54 Hauert C, De Monte S, Sigmund K, Hofbauer J: Oscillations in Optional Public Good Games. IIASA Interim Report IR-01-036 (2001).

No. 55 Ferrière R, Le Galliard J: Invasion Fitness and Adaptive Dynamics in Spatial Population Models. IIASA Interim Report IR-01-043 (2001). Clobert J, Dhondt A, Danchin E, Nichols J (eds): Dispersal, Oxford University Press, pp. 57-79 (2001).

No. 56 de Mazancourt C, Loreau M, Dieckmann U: Can the Evolution of Plant Defense Lead to Plant-Herbivore Mutualism. IIASA Interim Report IR-01-053 (2001). The American Naturalist 158: 109-123 (2001).

No. 57 Claessen D, Dieckmann U: Ontogenetic Niche Shifts and Evolutionary Branching in Size-Structured Populations. IIASA Interim Report IR-01-056 (2001). Evolutionary Ecology Research 4:189-217 (2002).

No. 58 Brandt H: Correlation Analysis of Fitness Landscapes. IIASA Interim Report IR-01-058 (2001).

No. 59 Dieckmann U: Adaptive Dynamics of Pathogen-Host Interacations. IIASA Interim Report IR-02-007 (2002). Dieckmann U, Metz JAJ, Sabelis MW, Sigmund K (eds): Adaptive Dynamics of Infectious Diseases: In Pursuit of Virulence Management, Cambridge University Press, Cambridge, UK, pp. 39-59 (2002).

No. 60 Nowak MA, Sigmund K: Super- and Coinfection: The Two Extremes. IIASA Interim Report IR-02-008 (2002). Dieckmann U, Metz JAJ, Sabelis MW, Sigmund K (eds): Adaptive Dynamics of Infectious Diseases: In Pursuit of Virulence Management, Cambridge University Press, Cambridge, UK, pp. 124-137 (2002).
No. 61 Sabelis MW, Metz JAJ: Perspectives for Virulence Management: Relating Theory to Experiment. IIASA Interim Report IR-02-009 (2002). Dieckmann U, Metz JAJ, Sabelis MW, Sigmund K (eds): Adaptive Dynamics of Infectious Diseases: In Pursuit of Virulence Management, Cambridge University Press, Cambridge, UK, pp. 379-398 (2002).

No. 62 Cheptou P, Dieckmann U: The Evolution of SelfFertilization in Density-Regulated Populations . IIASA Interim Report IR-02-024 (2002). Proceedings of the Royal Society of London Series B 269:1177-1186 (2002).

No. 63 Bürger R: Additive Genetic Variation Under Intraspecific Competition and Stabilizing Selection: A Two-Locus Study. IIASA Interim Report IR-02-013 (2002). Journal of Theoretical Population Biology 61:197-213 (2002).

No. 64 Hauert C, De Monte S, Hofbauer J, Sigmund K: Volunteering as Red Queen Mechanism for Co-operation in Public Goods Games. IIASA Interim Report IR-02-041 (2002). Science 296:1129-1132 (2002).

No. 65 Dercole F, Ferrière R, Rinaldi S: Ecological Bistability and Evolutionary Reversals under Asymmetrical Competition. IIASA Interim Report IR-02-053 (2002). Evolution 56:1081-1090 (2002).

No. 66 Dercole F, Rinaldi S: Evolution of Cannibalistic Traits: Scenarios Derived from Adaptive Dynamics. IIASA Interim Report IR-02-054 (2002).

No. 67 Bürger R, Gimelfarb A: Fluctuating Environments and the Role of Mutation in Maintaining Quantitative Genetic Variation. IIASA Interim Report IR-02-058 (2002). Genetical Research 80:31-46 (2002).

No. 68 Bürger R: On a Genetic Model of Intraspecific Competition and Stabilizing Selection. IIASA Interim Report IR02-062 (2002)

No. 69 Doebeli M, Dieckmann U: Speciation Along Environmental Gradients. IIASA Interim Report IR-02-079 (2002). Nature 421:259-264 (2003).

No. 70 Dercole F, Irisson J, Rinaldi S: Bifurcation Analysis of a Prey-Predator Coevolution Model. IIASA Interim Report IR-02-078 (2002).

Issues of the IIASA Studies in Adaptive Dynamics series can be obtained at www.iiasa.ac.at/Research/ADN/Series.html or by writing to adn@iiasa.ac.at. 


\section{Contents}

1 Introduction 1

2 The canonical equation of monomorphic evolutionary dynamics 2

3 A model of prey-predator coevolution 5

4 Bifurcation analysis $\quad 7$

5 Discussion and conclusions $\quad 11$

6 Appendix 14 


\begin{abstract}
We show in this paper how numerical bifurcation analysis can be used to study the evolution of genetically transmitted phenotypic traits. For this, we consider the standard Rosenzweig-MacArthur prey-predator model and, following the so-called Adaptive Dynamics approach, we derive from it a second-order evolutionary model composed of two ODEs, one for the prey trait and one for the predator trait. Then, we perform a detailed bifurcation analysis of the evolutionary model with respect to various environmental and demographic parameters. Surprisingly, the evolutionary dynamics turn out to be much richer than the population dynamics. Up to three evolutionary attractors can be present and the bifurcation diagrams contain numerous global bifurcations and codimension-2 bifurcation points. Interesting biological properties can be extracted from these bifurcation diagrams. In particular, one can conclude that evolution of the traits can be cyclic and easily promote prey species diversity.
\end{abstract}




\author{
About the Authors \\ Fabio Dercole \\ CIRITA, Politecnico di Milano \\ Via Ponzio 34/5, 20133 Milano, Italy \\ Jean-Olivier Irisson \\ Ecole Normale Supérieure \\ 45 rue d'Ulm, 75005 Paris, France \\ Sergio Rinaldi \\ CIRITA, Politecnico di Milano \\ Via Ponzio 34/5, 20133 Milano, Italy \\ and \\ Adaptive Dynamics Network \\ International Institute for Applied Systems Analysis \\ A-2361 Laxenburg, Austria
}

\title{
Acknowledgement
}

The authors are grateful to Ulf Dieckmann, who invited them to participate in the Adaptive Dynamics Network program at the International Institute for Applied Systems Analysis, Laxenburg, Austria. The support of CESTIA (CNR, Milano, Italy) to S. Rinaldi is also acknowledged. 


\title{
Bifurcation Analysis of a Prey-Predator Coevolution Model
}

\author{
Fabio Dercole \\ Jean-Olivier Irisson \\ Sergio Rinaldi
}

\section{Introduction}

One of the most important notions in biology, namely evolution, is now recognized to be of primary importance in many fields of science. Evolution of markets, institutions, technologies, languages, and social rules are relevant examples. Thus, a well founded mathematical theory of evolution is now needed more than ever.

Evolving systems are in general composed of $N$ homogeneous subsystems identified by two features: dimension $n_{i}$ and characteristic trait $x_{i}$. For example, in ecology, the subsystems are interacting plant and animal populations, $n_{i}$ is the number of individuals of each population or, equivalently, the density of the population, and $x_{i}$ is a genetically transmitted phenotypic trait (e.g., body size). Both features vary in time, but densities can vary at a much faster rate than traits. This means that an evolving system has two distinct timescales: one is fast and concerns only the densities, that vary while traits remain practically constant, and the other concerns the slow variation of the traits entraining slow variations of the densities. In some favorable cases, these slow variations of the traits can be described by a standard ODE model, called evolutionary model.

The theoretical work developed so far has shown that evolutionary dynamics can be as complex as one can imagine. For example, cyclic regimes (7) (called Red Queen dynamics as in (27)) and chaotic regimes (3) are possible as well as evolutionary suicides and murders, which occur when the variation of the trait of a population entails the extinction of the same or another population $(20 ; 10)$. Moreover, an evolving system can also have alternative evolutionary attractors, in which case the fate of the system is determined by its ancestral conditions.

Once an evolutionary model is available, the powerful machinery of numerical bifurcation analysis can be applied to it. This is mandatory if the aim is to detect the impact of some strategic parameters on the evolution of the system. Systematic bifurcation analysis with respect to key environmental parameters could, for example, explain why ecosystems differ at various latitudes, altitudes, and depths. The few bifurcation studies performed to date on evolutionary models (see, for example, $(19 ; 21 ; 7))$ are far from satisfactory: they are inaccurate because they have been mainly carried out through simulation and they are incomplete because they refer to non-generic cases or point out only some aspects of the full bifurcation diagram. In this article we therefore present an accurate and detailed bifurcation analysis of a typical evolutionary model. The problem we tackle is the coevolution of prey and predator traits, a subject that has received a great deal of attention in the last decade (see (1) for a review). We consider two populations (prey and predator), and two traits (one for each population), and the bifurcation analysis of the evolutionary model is performed with respect to pairs of parameters. The results we obtain are of rather limited biological value because they refer to a specific prey-predator coevolution model. However, the methodology is very general and could be applied to other models in order to obtain, 
through a suitable comparative analysis, general conclusions on the coevolution of prey-predator communities.

The paper is organized as follows. In the next section we recall how, under suitable assumptions on the mutation and selection processes, a canonical evolutionary model can be derived from a general population model $(6 ; 5)$. Then, we focus on the well known Rosenzweig-MacArthur prey-predator model (26), showing how the canonical evolutionary model can be explicitly derived from it. Finally, we present the bifurcation analysis of the evolutionary model, and demonstrate how interesting biological conclusions can be extracted from it. Some comments and comparisons with the literature close the paper.

\section{The canonical equation of monomorphic evolutionary dynamics}

Consider two interacting populations, from now on called prey and predator populations, with densities $n_{1}$ and $n_{2}$ and phenotypic traits $x_{1}$ and $x_{2}$.

At ecological timescale (fast dynamics), the traits are constant while the densities vary according to two ODEs of the form:

$$
\begin{aligned}
& \dot{n}_{1}=n_{1} F_{1}\left(n_{1}, n_{2}, x_{1}, x_{2}\right) \\
& \dot{n}_{2}=n_{2} F_{2}\left(n_{1}, n_{2}, x_{1}, x_{2}\right)
\end{aligned}
$$

where $F_{i}$ is the net per capita growth rate of the $i$-th population. In the following, model (1), called resident model, is assumed to have one strictly positive and globally stable equilibrium $\bar{n}\left(x_{1}, x_{2}\right)$ for each $\left(x_{1}, x_{2}\right)$ belonging to a set of the trait space called stationary coexistence region. This condition is not strictly necessary, but it simplifies the discussion.

At evolutionary timescale (slow dynamics), the traits vary according to two ODEs called evolutionary model

$$
\begin{aligned}
& \dot{x}_{1}=k_{1} G_{1}\left(x_{1}, x_{2}\right) \\
& \dot{x}_{2}=k_{2} G_{2}\left(x_{1}, x_{2}\right)
\end{aligned}
$$

where $k_{1}$ and $k_{2}$ are suitable constant parameters determined by size and frequency of mutations. However, population densities vary slowly with the traits because, at evolutionary timescale, model (1) is always at the equilibrium $\bar{n}\left(x_{1}, x_{2}\right)$.

Some authors discuss evolutionary problems by assigning particular forms to the functions $G_{1}$ and $G_{2}$ in model (2) without connecting them with a population model (see (2) and references therein). More frequently, model (2) is derived from model (1) through various arguments $(16 ; 2)$. This is a little surprising, since the dynamics of the traits should reflect the characteristics of the mutation and selection processes, that, however, are not included in the resident model (1). In fact, the most transparent approach for deriving the evolutionary model (2) is the so-called Adaptive Dynamics approach $(15 ; 22 ; 6 ; 13 ; 12)$ based on the resident-mutant models, which describe the interactions among three populations, namely the two resident populations, and a mutant population with trait $x_{1}^{\prime}$ or $x_{2}^{\prime}$ (notice that this approach rules out the possibility that prey and predator mutants are present at the same time). When the prey population is split into two subpopulations (resident and mutant) with densities $n_{1}$ and $n_{1}^{\prime}$ and traits $x_{1}$ and $x_{1}^{\prime}$, the model is:

$$
\begin{aligned}
& \dot{n}_{1}=n_{1} f_{1}\left(n_{1}, n_{2}, n_{1}^{\prime}, x_{1}, x_{2}, x_{1}^{\prime}\right) \\
& \dot{n}_{2}=n_{2} f_{2}\left(n_{1}, n_{2}, n_{1}^{\prime}, x_{1}, x_{2}, x_{1}^{\prime}\right) \\
& \dot{n}_{1}^{\prime}=n_{1}^{\prime} f_{1}^{\prime}\left(n_{1}, n_{2}, n_{1}^{\prime}, x_{1}, x_{2}, x_{1}^{\prime}\right)
\end{aligned}
$$

The initial value of $n_{1}^{\prime}$ in these equations is very small because a mutant population is initially composed of one or a few individuals. A similar third order model involving the mutant trait $x_{2}^{\prime}$, the density $n_{2}^{\prime}$, and the function $f_{2}^{\prime}$ describes the case in which the mutant is a predator. In the ecological literature, models like model (3) are often called "competition models" because they 
describe the competition between two similar populations. Obviously, model (3), together with its companion model for the predator mutation, contains more information than the resident model (1). Indeed, the latter can be immediately derived from the former by disregarding the mutant equation and letting $n_{1}^{\prime}=n_{2}^{\prime}=0, x_{1}^{\prime}=x_{1}$ and $x_{2}^{\prime}=x_{2}$, thus obtaining:

$$
F_{i}\left(n_{1}, n_{2}, x_{1}, x_{2}\right)=f_{i}\left(n_{1}, n_{2}, 0, x_{1}, x_{2}, x_{i}\right)
$$

The functions $f_{i}$ and $f_{i}^{\prime}$, identifying the right-hand-sides of the resident-mutant models, are called fitness functions, and they enjoy a number of structural properties. Function $f_{1}$ satisfies the condition:

$$
f_{1}\left(n_{1}, n_{2}, n_{1}^{\prime}, x_{1}, x_{2}, x_{1}\right)=F_{1}\left(n_{1}+n_{1}^{\prime}, n_{2}, x_{1}, x_{2}\right)
$$

because, when $x_{1}=x_{1}^{\prime}$, resident and mutant individuals do not differ, so that only the total number of prey $\left(n_{1}+n_{1}^{\prime}\right)$ matters. Of course, the same property holds for the functions $f_{2}, f_{1}^{\prime}$, and $f_{2}^{\prime}$. Another structural property of the fitness functions is:

$$
f_{1}^{\prime}\left(n_{1}, n_{2}, n_{1}^{\prime}, x_{1}, x_{2}, x_{1}^{\prime}\right)=f_{1}\left(n_{1}^{\prime}, n_{2}, n_{1}, x_{1}^{\prime}, x_{2}, x_{1}\right)
$$

because any one of the two prey subpopulations can be considered as mutant, provided the other is considered as resident.

Now that we have defined the resident model (1) and the resident-mutant model (3), we can show how the evolutionary model (2) can be derived following the Adaptive Dynamics approach. For this, assume that the resident population model (1) with traits $x_{1}$ and $x_{2}$ is at its equilibrium $\bar{n}\left(x_{1}, x_{2}\right)$ when a mutant appears. If mutations are rare at ecological timescale, the initial conditions $\left(\bar{n}_{1}\left(x_{1}, x_{2}\right), \bar{n}_{2}\left(x_{1}, x_{2}\right), n_{1}^{\prime}\right)$ can be used in model (3) to determine the fate of the mutant population. If the mutant population does not invade, i.e., if:

$$
f_{1}^{\prime}\left(\bar{n}_{1}\left(x_{1}, x_{2}\right), \bar{n}_{2}\left(x_{1}, x_{2}\right), n_{1}^{\prime}, x_{1}, x_{2}, x_{1}^{\prime}\right)<0
$$

for all small $n_{1}^{\prime}>0$, then it becomes extinct and the final result is still a pair of resident populations with traits $x_{1}$ and $x_{2}$ and densities $\bar{n}_{1}\left(x_{1}, x_{2}\right)$ and $\bar{n}_{2}\left(x_{1}, x_{2}\right)$. By contrast, it can be proved (11) that if (4) holds with the opposite inequality sign and if mutations are small (i.e., $x_{1}^{\prime}$ differs only slightly from $x_{1}$ ), then the resident population generically becomes extinct and is replaced by the mutant population with density $\bar{n}_{1}\left(x_{1}^{\prime}, x_{2}\right)$. In other words, each mutation brings a new trait into the system, but competition between resident and mutant populations selects the winner, namely the trait that remains in the system. This kind of evolution of the traits is called monomorphic evolution. This process of mutation and selection can be further specified by making suitable assumptions on the frequency and distribution of small mutations $(6 ; 5)$, and the conclusion is that the rate at which the trait $x_{i}$ varies at evolutionary timescale is given by the following ODE, called canonical equation of Adaptive Dynamics:

$$
\dot{x}_{i}=k_{i} \bar{n}_{i}\left(x_{1}, x_{2}\right) \frac{\partial f_{i}^{\prime}}{\partial x_{i}^{\prime}}\left|\begin{array}{l}
n_{1}=\bar{n}_{1}\left(x_{1}, x_{2}\right) \\
n_{2}=\bar{n}_{2}\left(x_{1}, x_{2}\right) \\
n_{i}^{\prime}=0 ; x_{i}^{\prime}=x_{i}
\end{array}\right|
$$

where $k_{i}$ is proportional to the frequency and variance of mutations, $\bar{n}_{i}\left(x_{1}, x_{2}\right)$ is the equilibrium density of the resident model, and $\partial f_{i}^{\prime} / \partial x_{i}^{\prime}$ is the derivative of the fitness of the mutant, called selective derivative. Equation (5), written for the prey and for the predator, gives two ODEs that form the evolutionary model (2) with:

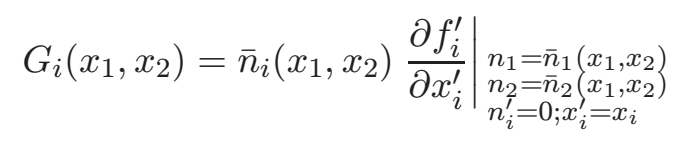




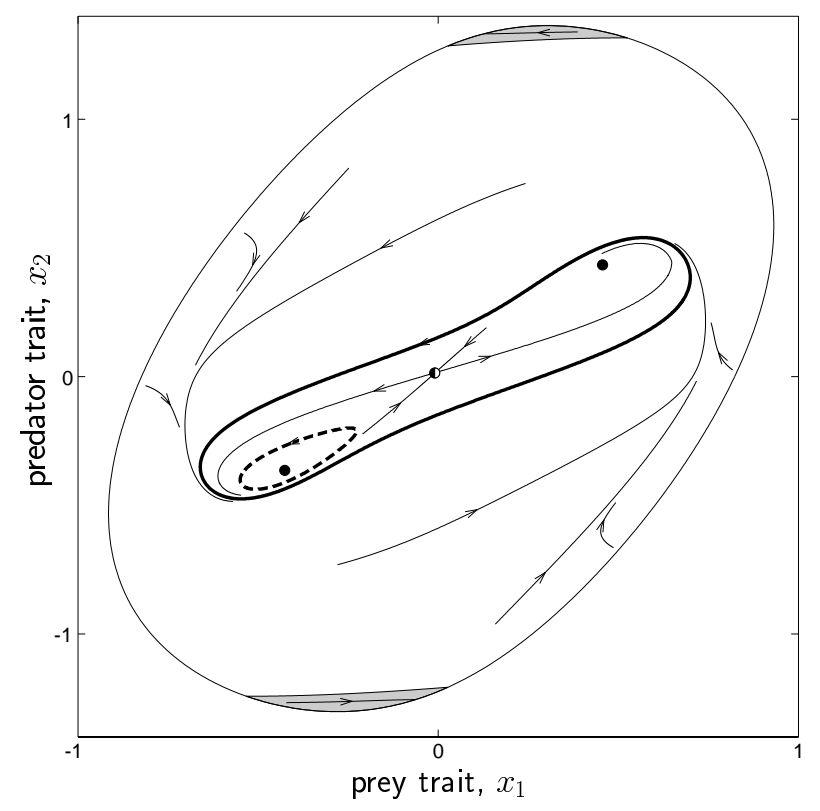

Figure 1: Example of coevolutionary portrait in the stationary coexistence region. The portrait is characterized by three equilibria (two stable foci (filled circles) and one saddle (half-filled circle)) and two limit cycles (one stable (thick line) and one unstable (dashed line)).

Thus, model (5) describes the monomorphic coevolution of the traits under the assumption of rare and random mutations of small effects. Monomorphic evolutionary dynamics are usually presented by drawing a few trajectories of model (5) in the stationary coexistence region. This set of trajectories, called coevolutionary portrait, points out, as sketched in Figure 1, all relevant invariant sets (equilibria, limit cycles, and saddle separatrices). Some trajectories of the coevolutionary portrait (see gray regions in Figure 1) reach the boundary of the stationary coexistence region, thus implying the extinction of one of the two populations.

Figure 2 schematically summarizes monomorphic evolution, and highlights the different roles played by the three models we have introduced. The ecological literature mainly deals with the resident model (1) since ecologists are interested in the short-term dynamics of the populations and usually do not even consider the possibility of having a mutant population involved in the game. By contrast, theoretical studies on evolution are based on formal evolutionary models (2), or on verbal theories that can be considered as a sort of surrogate of these models. Figure 2 points out two facts. The first is that both the resident model (1) and the evolutionary model (2) are needed if one is interested in the population dynamics entrained at evolutionary timescale by the dynamics of the traits. The second is that the resident-mutant model (3) is a "source" model, namely a model that contains the information needed to answer all questions. Unfortunately, the scheme of Figure 2 is not always taken into account and evolutionary models (2) are often derived directly from the resident model through arguments, which at best give the same result that a hidden equivalent resident-mutant model would give.

Once monomorphic dynamics has found a halt at a stable monomorphic equilibrium $\bar{x}$, one can look at the second-order terms in the Taylor expansion of the mutant fitness function to establish if the equilibrium is a branching point (13) or not. More precisely, a stable equilibrium $\bar{x}$ is said to be a branching point if

$$
\left.\frac{\partial^{2} f_{i}^{\prime}}{\partial x_{i}^{\prime 2}}\right|_{\substack{n_{1}=\bar{n}_{1}\left(\bar{x}_{1}, \bar{x}_{2}\right) \\ n_{2}=\bar{n}_{2}\left(\bar{x}_{1}, \bar{x}_{2}\right) \\ n_{i}^{\prime}=0 ; x_{i}^{\prime}=x_{i} ; x=\bar{x}}}>0
$$




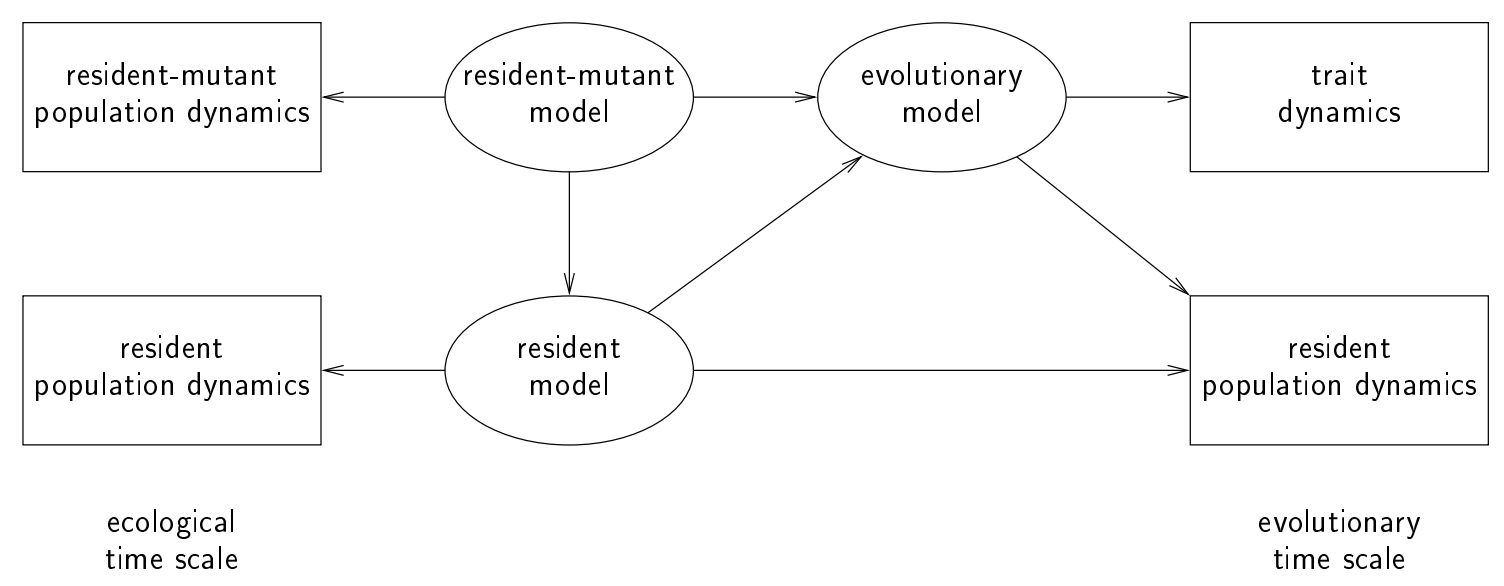

Figure 2: Flow chart demonstrating the relationships among resident-mutant models, resident model and evolutionary model.

and

$$
\frac{\partial^{2} f_{i}^{\prime}}{\partial x_{i}^{\prime} \partial x_{i}} \mid \begin{aligned}
& n_{1}=\bar{n}_{1}\left(\bar{x}_{1}, \bar{x}_{2}\right) \\
& \left.n_{2}=\bar{n}_{2} \bar{x}_{1}, \bar{x}_{2}\right) \\
& n_{i}^{\prime}=0 ; x_{i}^{\prime}=x_{i} ; x=\bar{x}
\end{aligned} \quad<0
$$

for $i$ equal to 1 or 2 , since small mutations of the $i$-th population invade and coexist, at equilibrium, with the former resident (12). Thus, branching points are the origin of dimorphism. Of course, after a branching has occurred, there are three resident populations and one can continue the analysis by deriving the three corresponding canonical equations.

\section{A model of prey-predator coevolution}

In this section we specify the prey-predator coevolution problem, which is analyzed in what follows. First we present the resident prey-predator model that has most often been used in the last few decades to predict prey and predator abundances at ecological timescale in the absence of mutations. We then extend this model to a scenario in which a mutant population is also present, by adding a third ODE for the mutant population and by specifying the dependence of the demographic parameters upon the traits of the resident and mutant populations. This produces a resident-mutant population model from which, following the scheme described in the previous section, we finally derive an evolutionary model of the form (2) (details are relegated in Appendix).

The population model we consider is the well known Rosenzweig-MacArthur prey-predator model (26)

$$
\begin{aligned}
& \dot{n}_{1}=n_{1}\left(r-c n_{1}-\frac{a}{1+a h n_{1}} n_{2}\right) \\
& \dot{n}_{2}=n_{2}\left(e \frac{a n_{1}}{1+a h n_{1}}-d\right)
\end{aligned}
$$

where $r$ is prey growth rate per capita, $c$ is prey intraspecific competition, $a$ is predator attack rate, $h$ is predator handling time, namely the time needed by each predator to handle and digest one unit of prey, $e$ is efficiency, namely a conversion factor transforming each unit of predated biomass into predator newborns, and $d$ is predator death rate. The reader interested in more details on the biological interpretation of the parameters can refer to (23). The six positive parameters of the model $(r, c, a, h, e, d)$ could be reduced to three through rescaling. However, we do not follow this option because it would complicate the biological interpretation of the dependence of 
the parameters upon the prey and predator traits. In order to have a meaningful problem one must assume that $e>d h$, because, otherwise, the predator population cannot grow even in the presence of an infinitely abundant prey population.

For any meaningful parameter setting, model (9) has a global attractor in $\mathbb{R}_{+}^{2}$, namely

(a) the trivial equilibrium $(r / c, 0)$ if $d / a(e-d h) \geq r / c$

(b) the strictly positive equilibrium

$$
\bar{n}_{1}=\frac{d}{a(e-d h)} \quad \bar{n}_{2}=\frac{c}{a}\left(\frac{r}{c}-\frac{d}{a(e-d h)}\right)\left(1+a h \frac{d}{a(e-d h)}\right)
$$

if

$$
\frac{r a h-c}{2 a h c} \leq \frac{d}{a(e-d h)}<\frac{r}{c}
$$

(c) a strictly positive limit cycle if $d / a(e-d h)<(r a h-c) /(2 a h c)$

The transition from $(a)$ to $(b)$ is a transcritical bifurcation (which is generic in the class of positive systems of the form (9)), while the transition from $(b)$ to $(c)$ is a supercritical Hopf bifurcation (see (17) for a proof).

If we now imagine that a mutant population is also present, we can enlarge model (9) by adding a third ODE and by slightly modifying the equations of the resident populations in order to take the mutant population into account. Of course we also need to specify how the parameters depend upon the traits $x_{1}, x_{2}, x_{1}^{\prime}, x_{2}^{\prime}$. The number of possibilities is practically unlimited, because even for well identified prey and predator species there are many meaningful options. Thus, at this level of abstraction, it is reasonable to limit the number of parameters sensitive to the traits and avoid trait dependencies that could give rise to biologically unrealistic evolutionary dynamics, like the unlimited growth of a trait (so-called runaway). Our choice has been to assume that the parameters $r, e$, and $d$ are independent of the traits, because this will allow us to compare our results with those obtained in (7). Thus, in the case of a mutation in the prey population, the resident-mutant model is

$$
\begin{aligned}
\dot{n}_{1} & =n_{1}\left(r-c\left(x_{1}, x_{1}\right) n_{1}-c\left(x_{1}, x_{1}^{\prime}\right) n_{1}^{\prime}+\right. \\
& \left.-\frac{a\left(x_{1}, x_{2}\right)}{1+a\left(x_{1}, x_{2}\right) h\left(x_{1}, x_{2}\right) n_{1}+a\left(x_{1}^{\prime}, x_{2}\right) h\left(x_{1}^{\prime}, x_{2}\right) n_{1}^{\prime}} n_{2}\right) \\
\dot{n}_{2} & =n_{2}\left(e \frac{a\left(x_{1}, x_{2}\right) n_{1}+a\left(x_{1}^{\prime}, x_{2}\right) n_{1}^{\prime}}{1+a\left(x_{1}, x_{2}\right) h\left(x_{1}, x_{2}\right) n_{1}+a\left(x_{1}^{\prime}, x_{2}\right) h\left(x_{1}^{\prime}, x_{2}\right) n_{1}^{\prime}}-d\right) \\
\dot{n}_{1}^{\prime} & =n_{1}^{\prime}\left(r-c\left(x_{1}^{\prime}, x_{1}\right) n_{1}-c\left(x_{1}^{\prime}, x_{1}^{\prime}\right) n_{1}^{\prime}+\right. \\
& \left.-\frac{a\left(x_{1}^{\prime}, x_{2}\right)}{1+a\left(x_{1}, x_{2}\right) h\left(x_{1}, x_{2}\right) n_{1}+a\left(x_{1}^{\prime}, x_{2}\right) h\left(x_{1}^{\prime}, x_{2}\right) n_{1}^{\prime}} n_{2}\right)
\end{aligned}
$$

The traits are assumed to be real variables obtained from the actual phenotypic traits through a suitable nonlinear scaling that maps the positive interval of the phenotype into the real axis. Thus, the maximum and minimum values of the prey [predator] phenotype correspond to the limit values $\infty$ and $-\infty$ of $x_{1}\left[x_{2}\right]$. Similarly, in the case of a mutation in the predator population, the 
resident-mutant model is

$$
\begin{aligned}
\dot{n}_{1} & =n_{1}\left(r-c\left(x_{1}, x_{1}\right) n_{1}+\right. \\
& \left.-\frac{a\left(x_{1}, x_{2}\right)}{1+a\left(x_{1}, x_{2}\right) h\left(x_{1}, x_{2}\right) n_{1}} n_{2}-\frac{a\left(x_{1}, x_{2}^{\prime}\right)}{1+a\left(x_{1}, x_{2}^{\prime}\right) h\left(x_{1}, x_{2}^{\prime}\right) n_{1}} n_{2}^{\prime}\right) \\
\dot{n}_{2} & =n_{2}\left(e \frac{a\left(x_{1}, x_{2}\right) n_{1}}{1+a\left(x_{1}, x_{2}\right) h\left(x_{1}, x_{2}\right) n_{1}}-d\right) \\
\dot{n}_{2}^{\prime} & =n_{2}^{\prime}\left(e \frac{a\left(x_{1}, x_{2}^{\prime}\right) n_{1}}{1+a\left(x_{1}, x_{2}^{\prime}\right) h\left(x_{1}, x_{2}^{\prime}\right) n_{1}}-d\right)
\end{aligned}
$$

The functional forms specifying the parameters dependence upon the traits are reported in Appendix and are such that the left inequality of condition (11) (i.e., $(r a h-c) /(2 a h c) \leq d /(a(e-$ $d h))$ ) is always satisfied (this excludes the possibility of population cycles). Thus, the boundary of the stationary coexistence region is simply the set of pairs $\left(x_{1}, x_{2}\right)$ for which $d /(a(e-d h))=r / c$ (see eq. (11)). This means that on that boundary $\bar{n}_{2}\left(x_{1}, x_{2}\right)=0$, i.e., the predator population becomes extinct if the traits reach the boundary of the stationary coexistence region.

At this point eq. (6) can be used to derive the evolutionary model (2), since the strictly positive equilibrium $\bar{n}\left(x_{1}, x_{2}\right)$ is known (see eq. (10)). The analytic expressions of the selective derivatives and of the second-order derivatives needed for evaluating the branching conditions $(7,8)$ are not reported because very long. In any case, they can be easily derived by means of any software for symbolic computation.

\section{Bifurcation analysis}

The evolutionary model derived in the previous section has been studied through numerical bifurcation analysis. Local and global codimension-1 bifurcations with respect to various parameters have been obtained by means of specialized software based on continuation techniques $(9 ; 8 ; 18)$. Moreover, two-dimensional bifurcation diagrams have been produced by focusing on codimension-2 bifurcation points (17).

The first surprising result is that the evolutionary model is much richer than the resident population model. In fact, while the latter is characterized by two bifurcations, 12 bifurcations have been detected in the former. Figure 3 shows these bifurcations in the space $(e, \gamma)$ where $e$ is predator efficiency and $\gamma$ is the prey trait value (called optimum) at which intraspecific competition is minimum. In general, both parameters are influenced by environmental factors. For example, the efficiency of a herbivore (predator) depends upon the caloric content of its prey (grass), which, in turn, is mainly fixed by humidity, temperature, and soil composition. Figure 3 points out that there are 14 subregions in the parameter space characterized by different coevolutionary portraits. In each one of them, for simplicity, the boundary of the stationary coexistence region, where the predator population becomes extinct, is not shown. This, however, fails to point out, graphically, that evolutionary extinction of the predator population occurs in all cases, as shown in Figure 1, which is actually the coevolutionary portrait corresponding to subregion 11. It is worth noticing that this form of evolutionary extinction is always an evolutionary murder. In fact, on the boundary of the stationary coexistence region $\dot{x}_{2}=0$, because $\bar{n}_{2}=0$ in eq. (5), i.e., the predator trait is locally constant while the prey trait varies.

Coevolutionary attractors can be equilibria or limit cycles and the existence of alternative attractors is rather common. When they exist, attracting cycles surround all equilibria. Actually, there can be up to three alternative attractors (two equilibria and one cycle), as shown by the coevolutionary portraits $10,11,13$, and 14 . There are 10 codimension- 2 bifurcation points, namely a cusp $(C)$, two generalized Hopf $\left(G H_{1}\right.$ and $\left.G H_{2}\right)$, two Bogdanov-Takens ( $B T_{1}$ and $\left.B T_{2}\right)$, four 


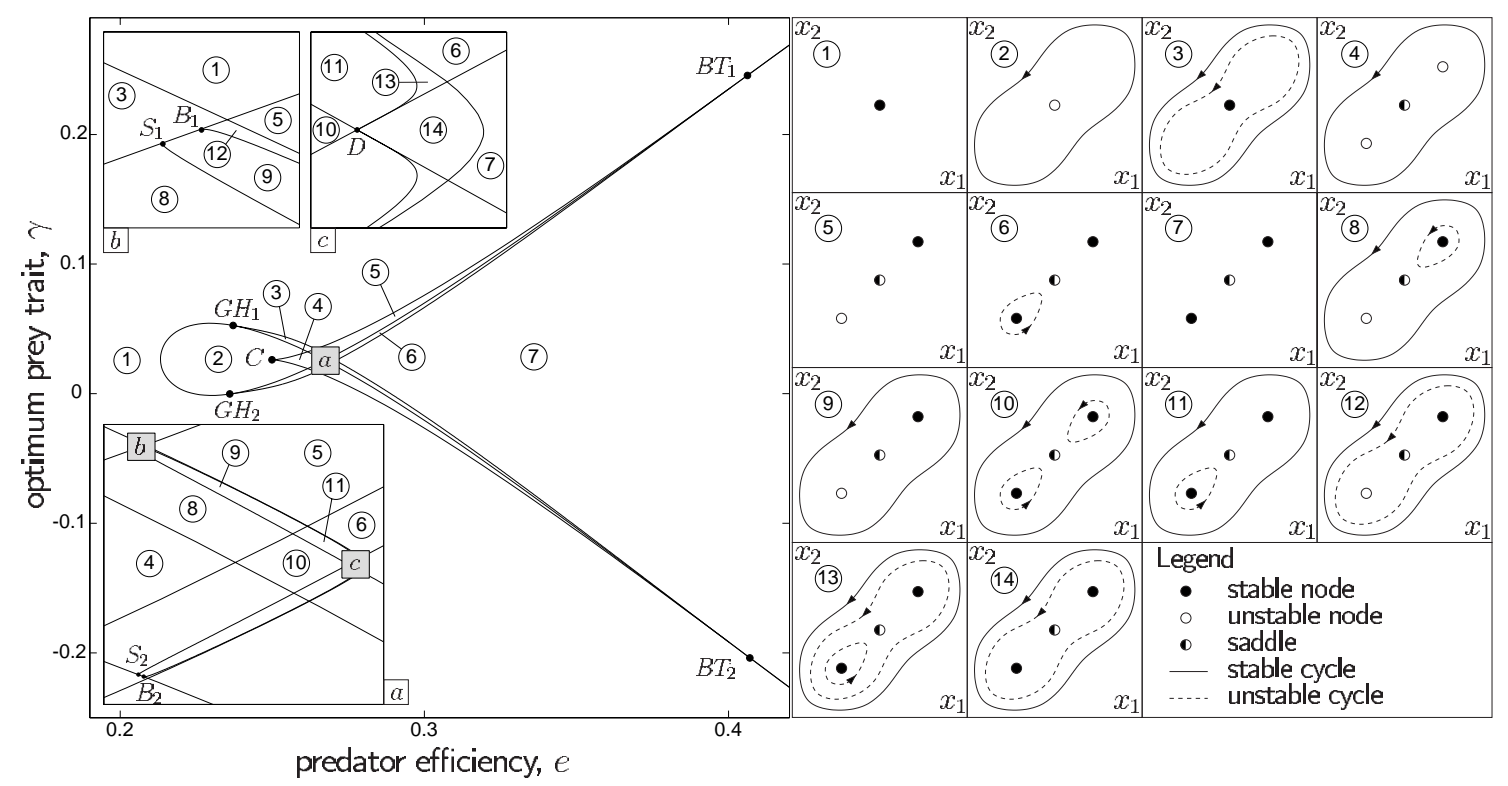

Figure 3: Bifurcation diagram of evolutionary model (5) with respect to predator efficiency $e$ and optimum prey trait $\gamma$ and corresponding sketches of coevolutionary state portraits. Panels $a, b$, and $c$ are magnified views of the bifurcation diagram. Parameter values are $r=0.5, d=0.05$, $k_{1}=k_{2}=1, \gamma_{0}=0.01, \gamma_{1}=0.5, \gamma_{2}=1, \alpha=1, \alpha_{0}=0.01, \alpha_{1}=1, \alpha_{2}=1, \alpha_{3}=0.6$, $\theta=0.9, \theta_{1}=\theta_{2}=0.5, \theta_{3}=\theta_{4}=1$.

non-central saddle-node homoclinic loops $\left(S_{1}, S_{2}, B_{1}\right.$, and $\left.B_{2}\right)$, and a double homoclinic loop (D) (17).

No other bifurcation curves and codimension- 2 bifurcation points are present in the two extra bifurcation diagrams presented in Figure 4, where the coevolutionary portraits are intentionally not shown to stress that they are exactly as in Figure 3. The parameter on the horizontal axis of these two bifurcation diagrams is still the efficiency of the predator, while the parameter on the vertical axis is related to two important characteristics of the mutation and predation processes, namely the ratio $k_{1} / k_{2}$ between the frequencies of prey and predator mutations and the predator handling time $\theta$ corresponding to the maximum attack rate (see Appendix).

The bifurcation diagrams are very useful for deriving interesting biological properties concerning the impact of various factors on coevolution. For example, one could be interested in identifying the factors favoring the so-called Red Queen dynamics, namely the possibility of cyclic coevolution of the traits. For this, one should extract from each bifurcation diagram the subregions $2-4,8-14$, where at least one of the coevolutionary attractors is a limit cycle. The result is Figure 5, which shows where cyclic coevolution is the only possible outcome (gray regions) and where stationary coevolution is also possible (black regions). Figure 5 indicates that Red Queen dynamics occur only for intermediate values of predator efficiency. Thus, slow environmental drifts entraining slow but continuous variations of predator efficiency can promote the disappearance of Red Queen dynamics. However, if efficiency decreases, Red Queen dynamics disappear smoothly through a supercritical Hopf bifurcation (where the attracting evolutionary cycle shrinks to a point). By contrast, if efficiency increases, Red Queen dynamics disappear discontinuously through a catastrophic bifurcation (tangent bifurcation of limit cycles). Figure 5 indicates also other biologically relevant properties, such as the fact that Red Queen dynamics are facilitated by high [low] frequency of prey [predator] mutation, and by low predator handling times. This last result shows that the highest chances for cyclic coevolution are obtained when $\theta=0$, i.e., 


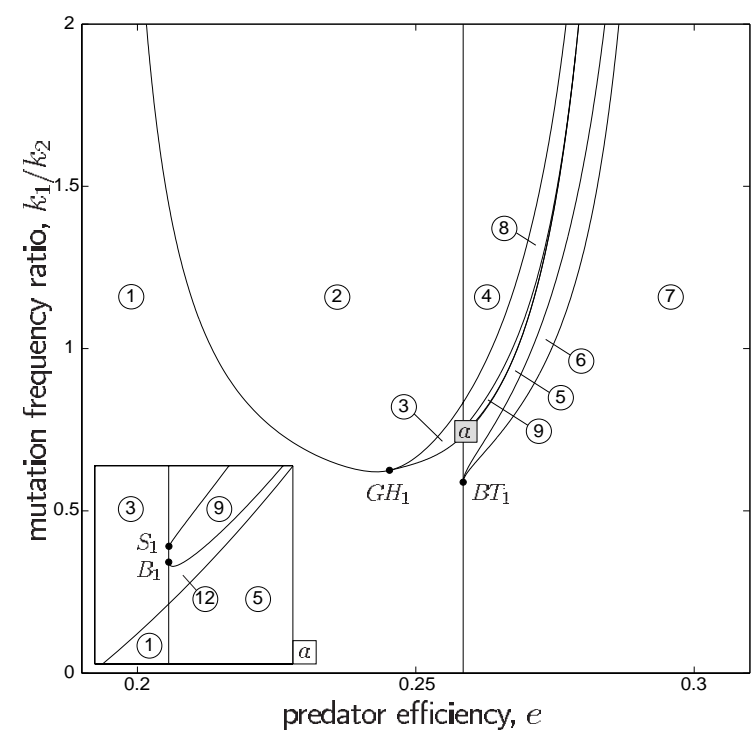

(A)

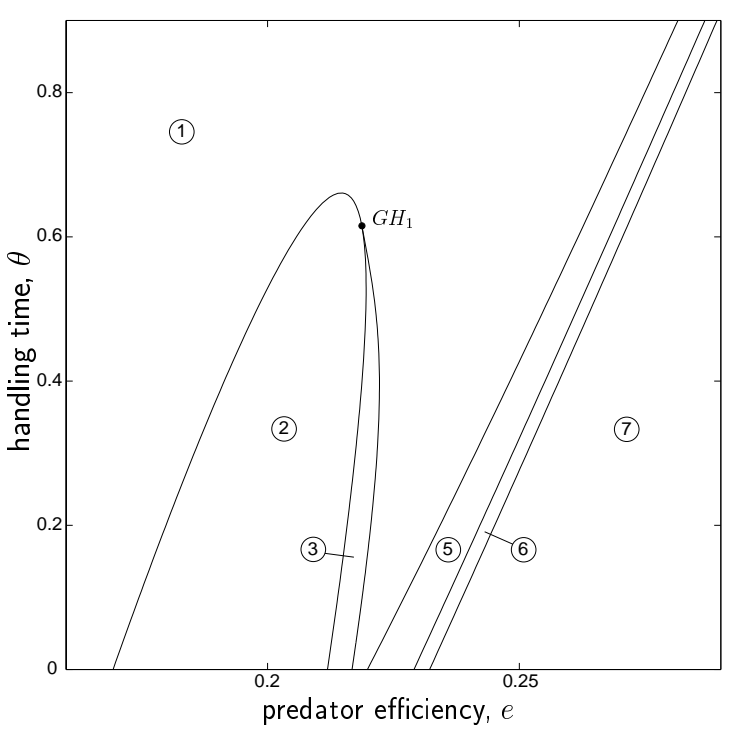

(B)

Figure 4: Bifurcation diagram of evolutionary model (5) with respect to predator efficiency $e$ and mutation frequency ratio $k_{1} / k_{2}(A)$ and handling time $\theta(B)$. See Figure 3 for coevolutionary state portraits and parameter values.

when the Rosenzweig-MacArthur model degenerates into the Lotka-Volterra model. This brings us to the following rather intriguing conclusion: the Lotka-Volterra assumptions (which do not give rise to population cycles) can easily explain coevolutionary cycles, while the RosenzweigMacArthur assumptions (which can easily give rise to population cycles) can hardly support Red Queen dynamics.

Extra information can be added to the bifurcation diagrams of Figures 3,4 by specifying if the stable monomorphic equilibria $\left(\bar{x}_{1}, \bar{x}_{2}\right)$ are branching points $(B)$ or not $(N B)$. This can be easily done by computing (through continuation) the curves where conditions $(7,8)$ are critical. Thus, any region of parameter space characterized by only one stable monomorphic equilibrium can, in principle, be partitioned into four subregions: in one of these subregions monomorphism is the only form of coevolution, while in the other three regions dimorphism is possible through the branching of one of the two populations or of both. However, in all the numerical experiments we have performed, only prey branching did occur. This is consistent with the well known principle of "competitive exclusion" (14). In fact, if predator would branch, the system would converge to an equilibrium with two slightly different predators and one prey, in contrast with the competitive exclusion principle. In conclusion, there are only two possibilities: $\left(\bar{x}_{1}, \bar{x}_{2}\right)$ is a branching point for the prey population or not. In other words, our findings are in line with biological principles and support the idea (4) that predators are promoters of prey species diversity. Figure 6 shows how the region characterized by a unique stable equilibrium $\left(\bar{x}_{1}, \bar{x}_{2}\right)$ is partitioned into $\mathrm{B}$ and NB subregions. The result is rather interesting if it is complemented with what has already been discovered about the disappearance of Red Queen dynamics induced by variations of predator efficiency. In fact, the overall conclusion is that Red Queen dynamics disappear abruptly if predator efficiency increases and smoothly if predator efficiency decreases. However, in the latter case, as soon as Red Queen dynamics disappear, dimorphism can occur in the prey population. Thus, environmental drifts of any sign can give rise to discontinuities in the dynamics of the traits. This observation proves, once more, that coevolution is an astonishingly complex dynamic process. 


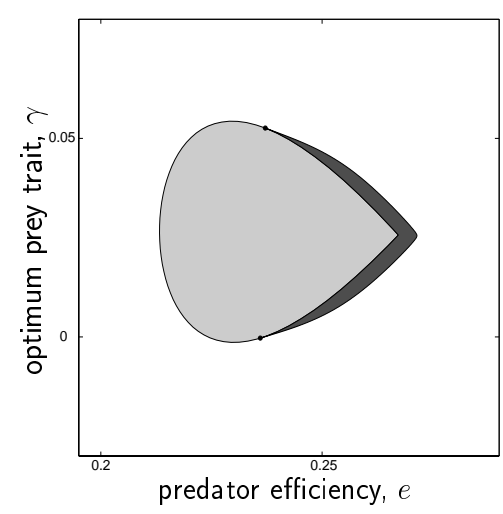

(A)

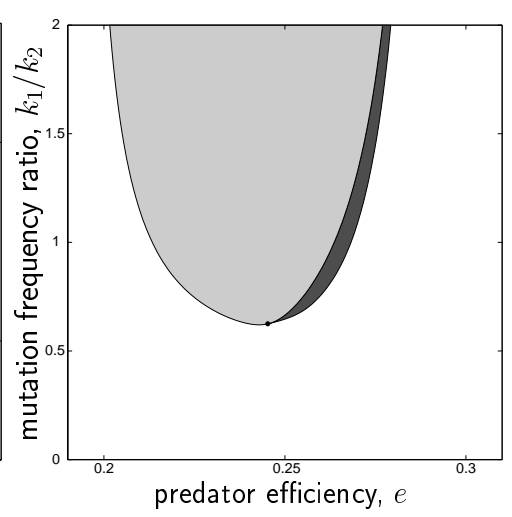

(B)

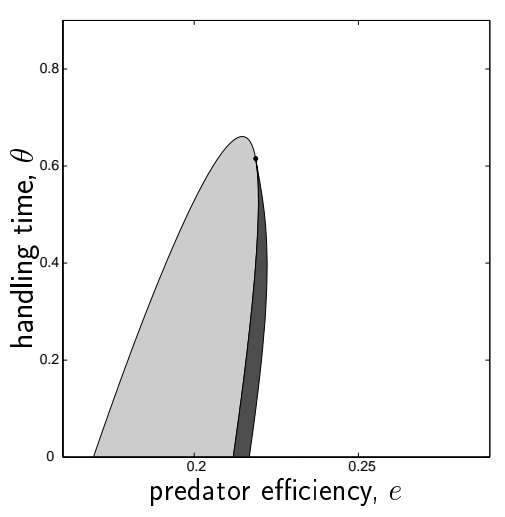

(C)

Figure 5: Red Queen dynamics: in the white regions cyclic coevolution is not possible, while in the gray regions it is the only long-term form of coevolution. In the black regions both stationary and cyclic coevolution are possible. Panels $(A),(B)$, and $(C)$ are extracted from the bifurcation diagrams of Figures 3, $4 A$, and $4 B$, respectively.

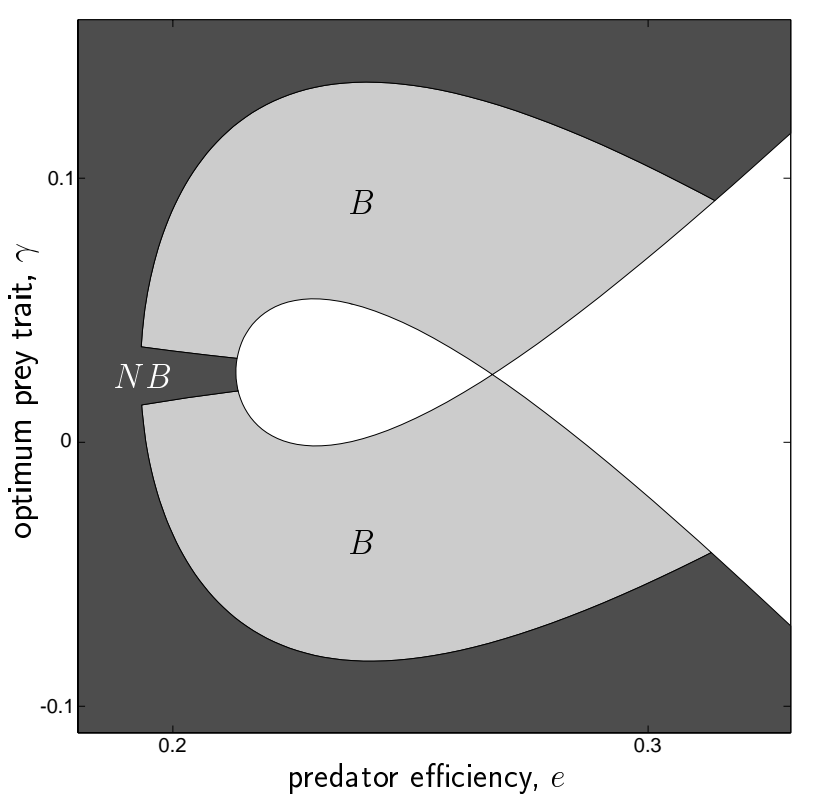

Figure 6: In the gray and black regions (extracted from Figure 3) the evolutionary model (5) has only one stable equilibrium, which is either a branching point (B) for the prey population or not (NB). 


\section{Discussion and conclusions}

The problem of prey-predator coevolution has been investigated in this paper from a purely mathematical point of view. For this, the classic Rosenzweig-MacArthur model (logistic prey and predator with saturating functional response) has first been transformed into a resident-mutant model by adding a third equation for the mutant population. Then, an evolutionary model describing the slow dynamics of the traits has been derived from the resident-mutant model through the standard Adaptive Dynamics approach $(15 ; 22 ; 6 ; 13 ; 12)$. The bifurcation analysis of the evolutionary model has shown that the dynamics of the traits at evolutionary timescale are much more complex than the dynamics of the populations at ecological timescale. The numerically produced bifurcation diagrams have proved to be powerful tools for extracting qualitative information on the impact of various factors on coevolution. Conclusions like those we have obtained on the impact of environmental drifts on evolutionary cycles (so-called Red Queen dynamics) could not have been derived without performing a detailed bifurcation analysis of an evolutionary model. A general encouraging message emerging from this study is that other very important biological problems, such as the evolution of mutualism, cannibalism, and parasitism, could most likely be studied successfully through the bifurcation analysis of the canonical evolutionary model. But the same approach should also be very effective for studying relevant problems in social sciences and economics where mechanisms somehow similar to biological mutation and selection can sometimes be identified.

Limiting the discussion to the problem of prey-predator coevolution, we can say that the results presented in this paper are by far much more complete than those available in the literature. Indeed, the only comparable result is the bifurcation analysis presented in (7) where a bifurcation diagram similar to that of Figure $4 A$ was obtained through simulation. That bifurcation diagram is incomplete and derived for a quite degenerate case, i.e. for a Lotka-Volterra model $(\theta=0$ in our model) with a very special parameter combination reducing the number of bifurcation curves to six. However, despite this double degeneracy, the analysis in (7) points out Red Queen dynamics, multiple evolutionary attractors, and evolutionary murder. A comparison with the nonmathematical literature (see, for example, $(24 ; 25)$ and (1)) neither contradicts nor supports our findings.

Even if what we have presented in this paper might seem rather general, the analysis should first be repeated for many other prey-predator models and for different assumptions on the traitdependence of the demographic parameters, and a comparative analysis should be performed in order to extract biologically significant results. Moreover, there are a number of possible interesting extensions. First, one could investigate the dynamics of dimorphism by applying the bifurcation approach followed in this paper to more complex population assemblies, composed, for example, of one resident predator population and two resident prey populations. The outcome of such a study could be that a predator branching generating a second resident predator population is possible, because this outcome is not in conflict with the principle of competitive exclusion. Second, while remaining in the simple context of monomorphism, one could be interested in detecting the prey-predator coevolutionary dynamics under the assumption that the two populations can coexist by cycling at ecological timescale. This extension is absolutely not trivial, because the derivation of the evolutionary model is rather difficult in this case. However, the problem is of great interest because its analysis could perhaps help to answer the very intriguing question: does coevolution destabilize populations? Third, one could be interested in extending the analysis to the coevolution of tritrophic food chains composed of a prey, a predator, and a superpredator population. From the results obtained in this paper, showing that evolutionary dynamics of ditrophic food chains (composed of a prey and a predator population) are much more complex than the corresponding population dynamics, one should naturally be inclined to conjecture that chaotic coevolutionary dynamics should be possible in tritrophic food chains. The proof of this conjecture would be a great result. 


\section{References}

[1] P. A. ABRAMS, The evolution of predator-prey interactions: Theory and evidence, Annual Review of Ecology and Systematics, 31 (2000), pp. 79-105.

[2] — Modelling the adaptive dynamics of traits involved in inter and intraspecific interactions: An assessment of three methods, Ecology Letters, 4 (2001), pp. 166-175.

[3] P. A. Abrams and H. Matsuda, Prey evolution as a cause of predator-prey cycles, Evolution, 51 (1997), pp. 1740-1748.

[4] J. S. BRown AND T. L. VincENT, Organization of predator-prey communities as an evolutionary game, Evolution, 46 (1992), pp. 1269-1283.

[5] N. Champagnat, R. Ferrière, AND G. Ben Arous, The canonical equation of adaptive dynamics: A mathematical view, Selection, 2 (2001), pp. 73-83.

[6] U. Dieckmann And R. LAw, The dynamical theory of coevolution: A derivation from stochastic ecological processes, Journal of Mathematical Biology, 34 (1996), pp. 579-612.

[7] U. Dieckmann, U. Marrow, AND R. LAW, Evolutionary cycling in predator-prey interactions: Population dynamics and the Red Queen, Journal of Theoretical Biology, 176 (1995), pp. 91-102.

[8] E. Doedel, A. Champneys, T. Fairgrieve, Y. A. Kuznetsov, B. Sandstede, And X. WANG, AUTO97: Continuation and bifurcation software for ordinary differential equations (with HOMCONT), Computer Science, Concordia University, Montreal, Canada, 1997.

[9] E. Doedel And J. P. Kernevez, AUTO: Software for continuation problems in ordinary differential equations, Applied Mathematics, California Institute of Technology, 1986.

[10] R. FERRIÈRE, Adaptive responses to environmental threats: Evolutionary suicide, insurance and rescue, Options, IIASA, Laxenburg, Austria,, Spring 2000 (2002), pp. 12-16.

[11] S. A. H. Geritz, M. Gyllenberg, F. J. A. Jacobs, and K. Parvinen, Invasion dynamics and attractor inheritance, Journal of Mathematical Biology, 44 (2002), pp. 548-560.

[12] S. A. H. Geritz, E. Kisdi, G. Meszéna, And J. A. J. Metz, Evolutionarily singular strategies and the adaptive growth and branching of the evolutionary tree, Ecology, 12 (1998), pp. 35-57.

[13] S. A. H. Geritz, J. A. J. Metz, E. Kisdi, And G. MeszénA, The dynamics of adaptation and evolutionary branching, Physical Review Letters, 78 (1997), pp. 2024-2027.

[14] G. HARDIn, The competitive exclusion principle, Science, 131 (1960), pp. 1292-1298.

[15] J. Hofbauer And K. Sigmund, Adaptive dynamics and evolutionary stability, Mathematical Letters, 3 (1990), pp. 75-79.

[16] A. I. Khibnik And A. S. Kondrashov, Three mechanisms of Red Queen dynamics, Proceedings of the Royal Society of London B, 264 (1997), pp. 1049-1056.

[17] Y. A. KuZnetsov, Elements of Applied Bifurcation Theory, Springer Verlag, Berlin, 1998.

[18] Y. A. Kuznetsov And V. V. LeVitin, CONTENT: A multiplatform environment for analyzing dynamical systems, Dynam. System Lab., Centrum voor Wiskunde en Inform., Amsterdam, available from ftp.cwi.nl/pub/CONTENT, 1997. 
[19] P. MARROW, R. LAW, AND C. CANNINGS, The coevolution of predator-prey interactions: ESSs and Red Queen dynamics, Proceedings of the Royal Society of London B, 250 (1992), pp. 133-141.

[20] H. Matsuda AND P. A. Abrams, Runaway evolution to self-extinction under asymmetrical competition, Evolution, 48 (1994), pp. 1764-1772.

[21] _ Timid consumers: Self extinction due to adaptive change in foraging and anti-predator effort, Theoretical Population Biology, 45 (1994), pp. 76-91.

[22] J. A. J. Metz, R. M. Nisbet, And S. A. H. Geritz, How should we define fitness for general ecological scenarios?, Trends in Ecology \& Evolution, 7 (1992), pp. 198-202.

[23] S. MURATORI AND S. RinALDI, Low- and high-frequency oscillations in three-dimensional food chain systems, SIAM Journal on Applied Mathematics, 52 (1992), pp. 1688-1706.

[24] D. PIMENTEL, Animal population regulation by the genetic feedback mechanism, The American Naturalist, 95 (1961), pp. 65-79.

[25] __, Population regulation and genetic feedback, Science, 159 (1968), pp. 1432-1437.

[26] M. L. RosenzWeIG AND R. H. MACARTHUR, Graphical representation and stability conditions of predator-prey interactions, The American Naturalist, 97 (1963), pp. 209-223.

[27] L. VAn VAlen, A new evolutionary law, Evolutionary Theory, 1 (1973), pp. 1-30. 


\section{Appendix}

In this appendix we specify how the prey intraspecific competition $c$, the predator attack rate $a$, and the predator handling time $h$, appearing in the resident-mutant models $(12,13)$, depend upon the resident and mutant traits. Due to our definition of the traits, which are scaled measures of the phenotypes, $c, a$, and $h$ are bounded functions of the traits. Unless otherwise stated, all the parameters appearing in these functions are assumed to be positive.

Prey intraspecific competition $c$ is given by

$$
c\left(x_{1}, x_{1}^{\prime}\right)=\frac{\gamma_{1}+\gamma_{2}\left(x_{1}-\gamma\right)^{2}}{1+\gamma_{0}\left(\gamma_{1}+\gamma_{2}\left(x_{1}-\gamma\right)^{2}\right)}
$$

Notice that $c$ depends only upon its first argument. This means that resident prey individuals face the same competition when they are opposed to other resident individuals or to mutant individuals. The parameter $\gamma$, which can be either positive or negative, is the value of the prey trait $x_{1}$ (called optimum prey trait) at which intraspecific competition is minimum (and equal to $\gamma_{1} /\left(1+\gamma_{0} \gamma_{1}\right)$ ). For prey traits $x_{1}$ far from $\gamma$ intraspecific competition saturates at $1 / \gamma_{0}$.

The predator attack rate $a$ is the bell-shaped function

$$
a\left(x_{1}, x_{2}\right)=\alpha_{0}+\alpha \exp \left(-\left(\frac{x_{1}}{\alpha_{1}}\right)^{2}+2 \alpha_{3}\left(\frac{x_{1}}{\alpha_{1}}\right)\left(\frac{x_{2}}{\alpha_{2}}\right)-\left(\frac{x_{2}}{\alpha_{2}}\right)^{2}\right)
$$

where $\alpha_{3}<1$. If prey and predator traits are tuned, i.e. if $x_{1}=x_{2}=0$, the predator attack rate is maximum (and equal to $\alpha_{0}+\alpha$ ). When prey and predator traits are far from being tuned, the predator attack rate drops to $\alpha_{0}$.

The predator handling time is the product of an increasing sigmoidal function of the prey trait $x_{1}$ and of a decreasing sigmoidal function of the predator trait $x_{2}$

$$
h\left(x_{1}, x_{2}\right)=\theta\left[1+\theta_{1}-\frac{2 \theta_{1}}{1+\exp \left(\theta_{3} x_{1}\right)}\right]\left[1+\theta_{2}-\frac{2 \theta_{2}}{1+\exp \left(-\theta_{4} x_{2}\right)}\right]
$$

where $\theta$ is the handling time corresponding to the tuned situation $\left(\left(x_{1}, x_{2}\right)=(0,0)\right)$, shortly called handling time in Figures $4 B$ and $5 C$.

Finally, we have fixed $r, d$ and all the parameters of the functions $c, a$, and $h$ at the values indicated in the caption of Figure 3, and we have limited $\theta$ from above and $e$ from below so that the following two inequalities hold for all $\left(x_{1}, x_{2}\right)$

$$
e-d h\left(x_{1}, x_{2}\right)>0 \quad \frac{r}{c\left(x_{1}\right)} \leq \frac{1}{a\left(x_{1}, x_{2}\right) h\left(x_{1}, x_{2}\right)}
$$

These conditions guarantee that the left inequality of condition (11) holds. Thus, population cycles are ruled out from our study. 